\title{
An evolutionary system of mineralogy: Employing mineral data to elucidate the co-evolution of the geo- and biosphere
}

\author{
ROBERT M. HAZEN \& SHAUNNA M. MORRISON
}

Earth \& Planets Laboratory, Carnegie Institution for Science, Washington DC 20015

Email: [rhazen@ciw.edu] [smorrison@carnegiescience.edu]

Information-rich attributes of minerals reveal their physical, chemical, and biological modes of origin in the context of planetary evolution. However, mineral classification by the Dana System relies on the minimum structural and chemical information necessary to distinguish $>5500$ species approved by the International Mineralogical Association (IMA: http://rruff.info/ima). Our "evolutionary system" of mineralogy embraces the multi-dimensional data-rich attributes of minerals that arise from their varied chemical, physical, and (since $>3.8 \mathrm{Ga}$ ) biological modes of formation [1-4]. We use cluster analysis to define mineral "natural kinds" based on combinations of attributes.

This evolutionary system differs from IMA protocols by distinguishing different mineral kinds using such revelatory attributes as trace and minor elements, fractionated isotopes, structural defects, mineral ages, and petrologic contextsattributes that provide powerful testimony regarding each mineral specimen's origins, as well as its subsequent deeptime interactions with changing geological environments. This system provides a framework for classifying minerals in the context of planetary evolution (Fig. 1).

The evolutionary system employs IMA nomenclature for most natural condensed solids, but it deviates from those protocols in three important ways: (1) In some instances, we split IMA species into two or more "natural kinds," based on diagnostic combinations of attributes that arise from distinct paragenetic modes. (2) In a number of cases, when a mineral displays a continuous range of compositions bounded by several different idealized end-members, we lump two or more approved IMA species into one natural kind. (3) We classify non-crystalline condensed phases that are not generally incorporated in the current IMA scheme.

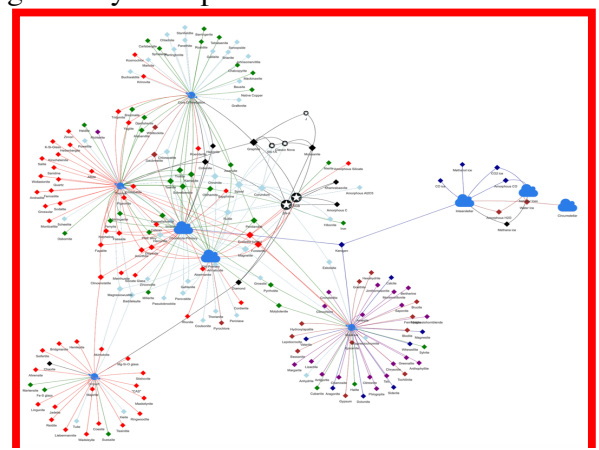

Fig. 1: Bipartite network graphs of early stages of mineral evolution reveal patterns in the diversity and distribution of minerals (Graph courtesy of Anirudh Prabhu, RPI).

1. R.M. Hazen (2014) Am. Mineral. 99, 2165-2170; 2. R.M. Hazen et al. (2019) China Engineering 5, 397-405; 3. R.M. Hazen (2019) Amer. Mineral. 104, 810-816; 4. R.M. Hazen \& S.M. Morrison (2020) Amer. Mineral. 105, in press. 